\title{
INVESTIGATION THE EFFECT OF USING GRAY LEVEL AND RGB CHANNELS ON BRAIN TUMOR IMAGE
}

\author{
Ahmed B Salem Salamh \\ Department of Computer, Zawia University, Zawia City, Libya
}

\begin{abstract}
Analysis the effect of using gray level on the Brain tumor image for improving speed of object detection in the field of Medical Image using image processing technique. Specific areas of interest are image binarization method, Image segmentation. Experiments will be performed by image processing using Matlab. This paper presents a strategy for decreasing the calculation time by using gray level and just one channel Red or Green or Blue in medical Image and analysis its impact in order to improve detection time and the main goal is to reduce time complexity.
\end{abstract}

\begin{abstract}
KEYWORDS
Image Segmentation, Medical Image, Detection Time, Brain Tumor image, Image Channels, Time Complexity
\end{abstract}

\section{INTRODUCTION}

Image processing is a very important field in the whole world these days and has become the necessary field of enhancing and analyze images. The image processing technique provides massive of methods to process and manipulate pictures in many fields, for example edge detection, image compression, facial recognition and medical image. However, image processing overcomes traditional use as a result of computers become more efficient and reliable. A computer is used to making a picture with different types as the properties of images that distinguish its extensions. On the other hand, using technique to alter and interpret real images. The main objectives of image processing are enhancing image quality and detection of information in order to clarify image objects in spite of sophisticated details that sometimes make it difficult to process. It is useful to use image processing methods as the first step to digitize a picture into an image file. The next step is applying some technique to enhance image quality. In addition, Image processing is growing very rapidly, for example, processing of medical image is one of the most important fields due to many reasons such as breast cancer and fracture bone detection, which causes lots of trouble in many years due to lack of research In a global term, image processing is one of computer applications which it uses many algorithms to process the image. Moreover, each algorithm has advantages and disadvantages that affect on image in different positions. Many applications have been developed in order to reach optimal technique that enable researchers to work more efficient and fast in this field. One of the important medical images is Magnetic resonance imaging (MRI). X-ray scanners utilize solid magnetic fields, radio waves, and field inclinations to produce pictures of the organs in the body. For instance, MRI is used to diagnose brain tumor in order to detect mass of abnormal tumor which is abnormal cell.

Dhinaharan Nagamalai et al. (Eds) : AIS, CSIT, IPPR, IPDCA - 2017

pp. 141- 148, 2017. (C) CS \& IT-CSCP 2017

DOI : $10.5121 /$ csit.2017.71012 
MRI makes it conceivable to introduce many sorts of tissue differentiate by changing excitation and reiteration times, which makes it an exceptionally adaptable instrument for imaging diverse structures of intrigue. Because of the nature and appearance of cerebrum tumors, one MRI arrangement is not adequate to completed portion the tumor including all its sub regions.

\section{RESEARCH PAPER OBJECTIVES}

This paper focuses on:

1- Analysis The effects of using gray level on Medical Image (MRI image) in order to get less information and improve time complexity and investigate the benefits of using gray level on the brain tumor medical image.

2- Experiments of techniques this paper use on different brain images, analysis, contrast and discuss the results.

3- Produce the conclusion.

\section{RELATED WORK}

Magnetic resonance imaging (MRI) ) is a fantastic medicnal imaging, especially for brain imaging. MRI inside the human body is useful to see the level of details. Specialists have real specialized and monetary significance of dependable also, quick detection and classification of brain malignancy, malignancy means in advanced stage. MRI is most proficient for the examination of brain tumor recognition and characterization when contrasted with other imaging procedures [1]. Brain tumor is a mass of tissue that grows out of control of the normal forces that regulates growth [2]. The Brain tumor is the most common, occurring malignancy among human beings [3]. The brain abnormality detection and segmentation of MRI images is an exceptionally hard to specify and crucial to assess which is utilized as a part of surgical and restorative arranging and appraisal. The trouble in brain picture examination is primarily because of the necessity of identification procedures with high exactness with speed time [4]. Brain tumor is diagnosed at advanced stages with the help of the MRI image [5]. Medical Image Analysis provides a forum for the dissemination information of new research results in the field of medical and biological image analysis, with special emphasis on efforts related to the applications of computer vision, virtual reality and robotics to biomedical imaging problems [6]. MRI image segmentation is an important but inherently difficult problem in medical image processing [7]. Image segmentation is used to separate objects from the background, and thus it has proved to be a powerful tool in bio-medical imaging. [8]. Tumor segmentation from magnetic resonance imaging (MRI) process is an important but time ingesting manual project performed by doctors. Magnetic resonance imaging (MRI), computed tomography (CT), virtual mammography, and other imaging strategy, supply an efficient way for detecting one-of-a-kind form of tumors[4]. In clinical photograph processing, the problems of reminiscence utilization and low execution velocity are compounded with ever-increasing sizes of information units. Normal high decision Computed Tomography (HRCT) photo sets now consist of masses of 512x512 slices, making up an (nearly) isotropic volume, that's quality treated as one volume for reasons of consistency of effects over the 0.33 axis [9].

In the medical field, it is still needed searching in this area, which is very important in the life because, it saves many people or enable specialized people to find out problems related to this as fast as possible. Many researchers have created image processing techniques to use it in specific system. This techniques has concentrated on advantage step of the process. For instance, using techniques such as an effecient segmentation algorithm by Fast scanning that was fast with the 
speed [10] but is not for abnormal region of brain image. There are several basic ways to segment any type of image. For example, Threshold Technique, Edge-Based Segmentation, Region-based Segmentation. .However, all previous method work with convert RGB image to grayscale as first step, which consist of value between 0 to 255 . From this is good to convert image to grayscale, but this paper proposes to process RGB image directly with extract of one color from the RGB image without converting images. Neeraja Menon and Rohit Ramakrishnan have proposed fast MRI Brain Image segmentation method based on Artificial Bee Colony $(\mathrm{ABC})$ [11]. The first step of their work, convert MRI image to grayscale.

P. S. Juhi and S. S. Kumar proposed Bounding box based automatic segmentation of brain tumors using random walker and active contours from brain MRI. They introduced fast and accurate segmentation method by segmenting the most dissimilar regions of a tumor image[12]. None of any algorithms used to process MRI image significantly with one of RGB colors of the original image.

\section{METHODS}

This section shows examples of some common image processing techniques that have been used in brain tumor for image display, analysis and human interpretation.

\section{Experiment Steps:}

1. Load color image from original MRI image.

2. Convert a gray image and read Red, Green and Blue image in order to manipulate each one.

3. Image binarization for each Red, Green and Blue image to select objects and observe the difference between the three images.

4. Histogram for each gray image, Red, Green and Blue image for clarification.

5. Object detection with circle for each Red, Green and Blue image.

The following flowchart shows in pictorial form how a method is finished from begin to the end in sequential step order. Moreover, represent the five steps with flowchart is needed in order to understand the main objective of the paper and it will clarify stages as it appear in figure 1 . 


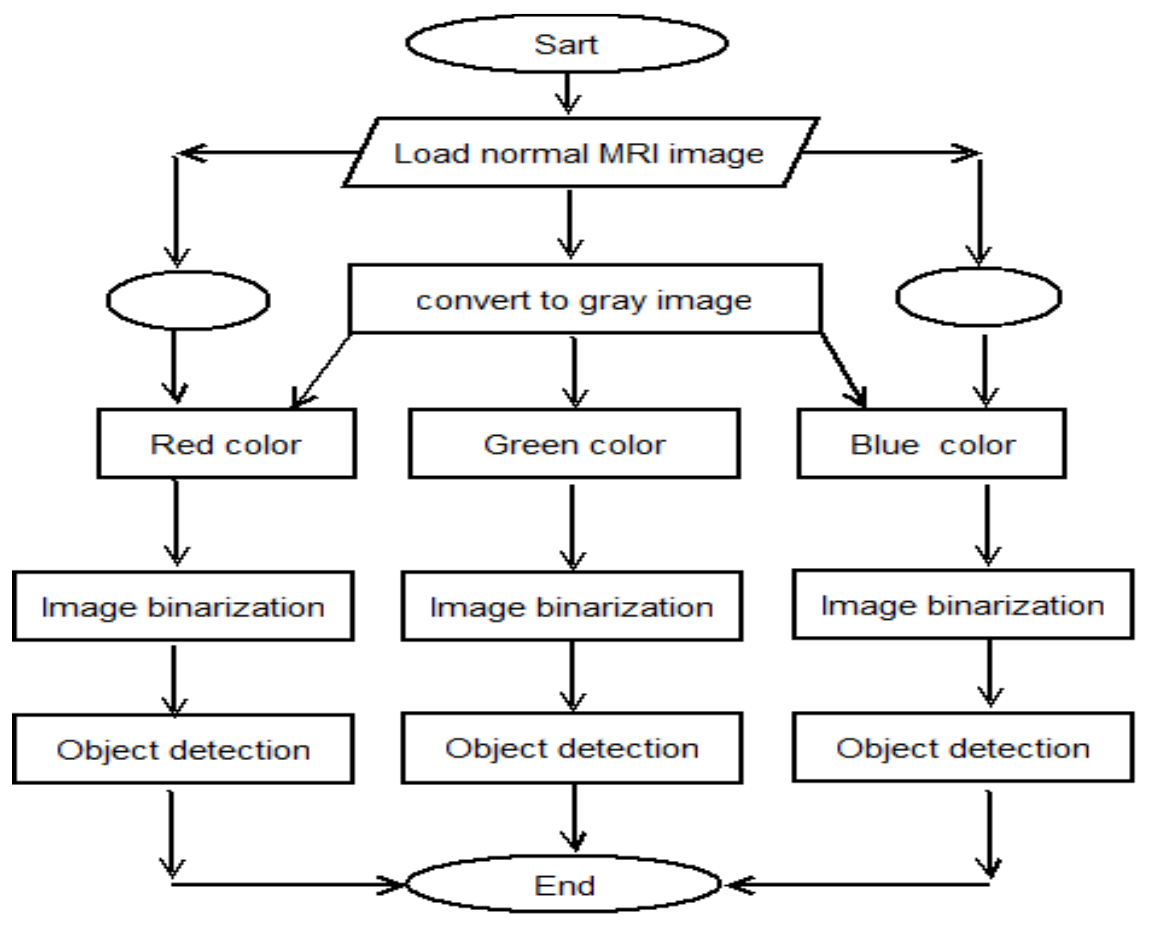

Figure 1. Clarification of experiment steps with different methods

\subsection{First experiment.}

In The first step, load the original MRI image with a brain tumor. The next step As we can see in the figure 2 convert MRI image to gray image in order to process images and makes the comparison.

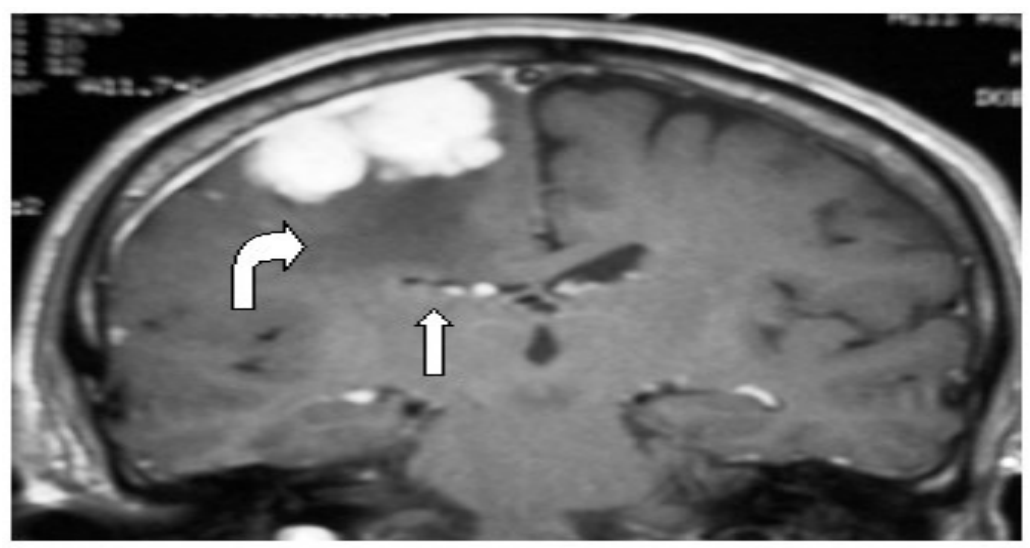

Figure 2. Presents gray level information from the original MRI image which captures brain tumor

The next part shows original red, green, blue colors of the image. In this part we have a choice to extract three colors from gray image or extract one channel from the original color image.

This paper uses the method to extract red, green, blue Sequentially and present them in order to apply segmentation and binarization on three colors as it appears in the following figure. 


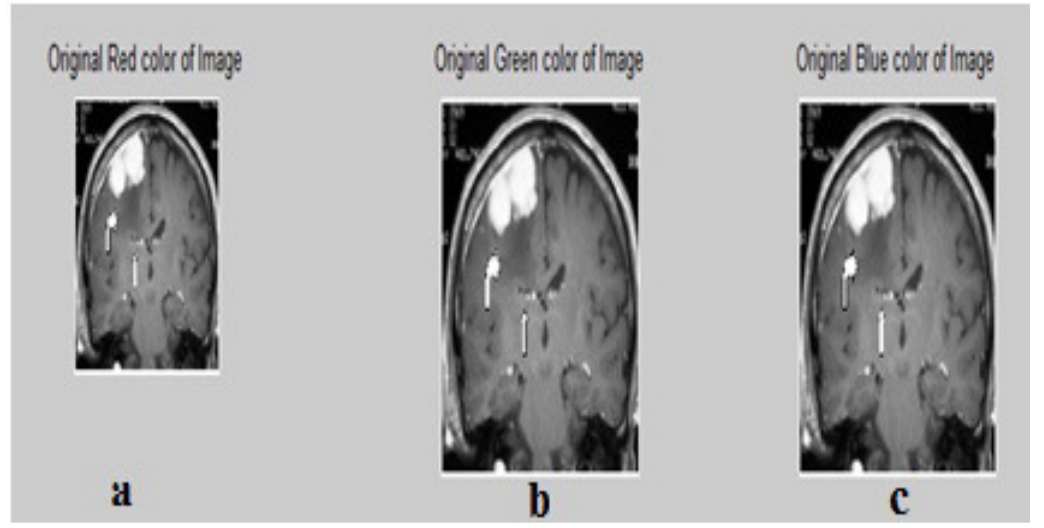

Figure 3. Separate gray image (a) Red (b) Green (c) Blue

The next section is applying segmentation and binarization and function to neglect the connected pixel in specified size in order to present object and detect brain tumor. Furthermore, this will apply on three colors sequentially to analysis the effect of object detection. This is clear in the figure 4.

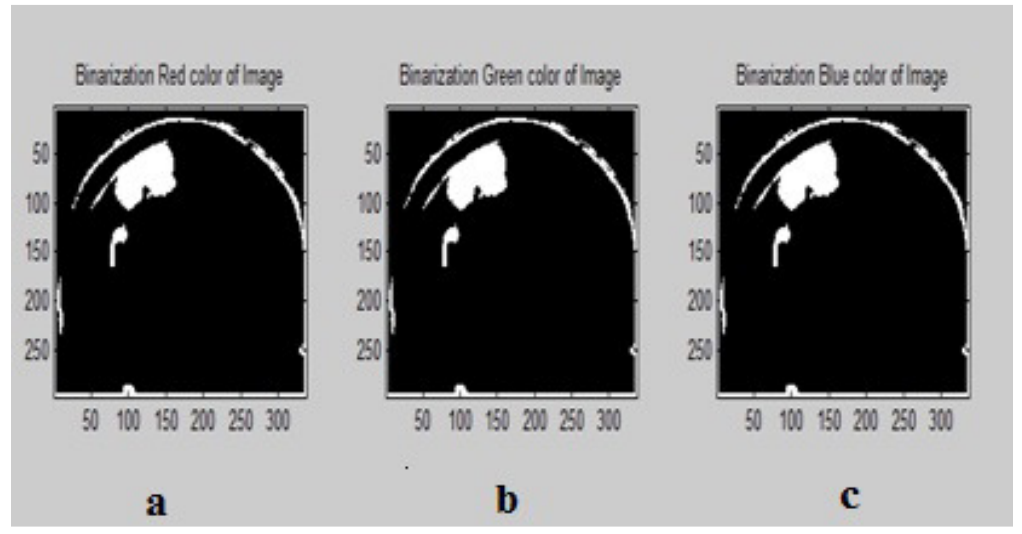

Figure 4. Presents image binarization (a) Red (b) Green (c) Blue image

The histogram is useful to check the a distribution of discrete intensity levels or the range of quantities in the specified graph. In the figure 5, it is clear to observe the distribution of one color in each part red, green, blue and its has the same distribution and its presented in figure 5.

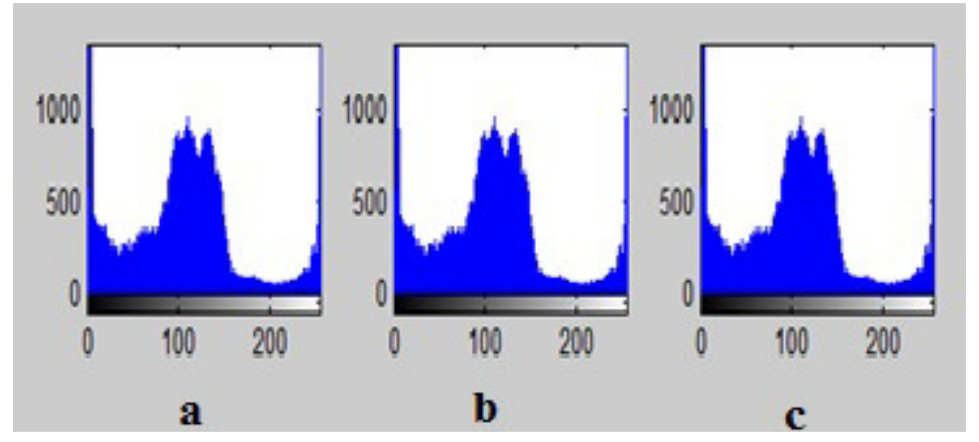

Figure 5. Presents histogram (a) Red (b) Green (c) Blue image. 
The histogram in the next figure is to grayscale image comes from the original color image to demonstrate the comparison of any color red or green or blue as a result of this there is no difference between them as it showed in figure 6 .

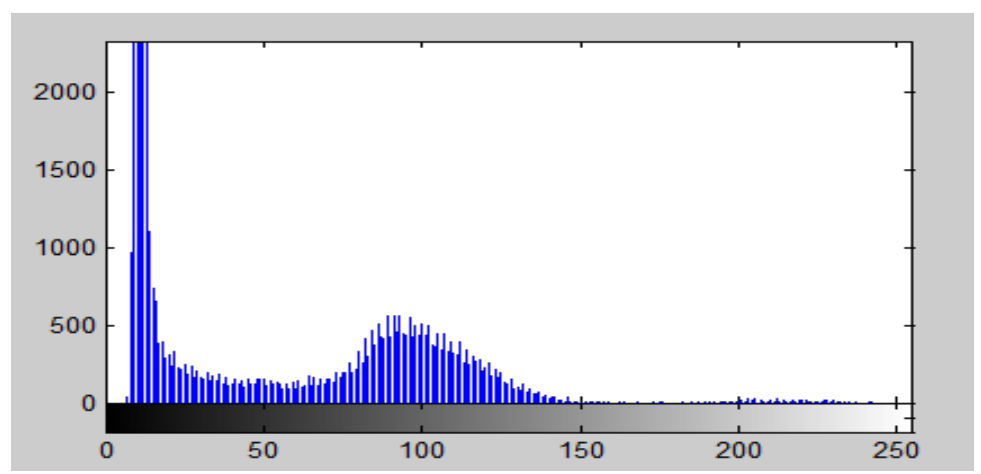

Figure 6. Presents histogram for the original gray image.

The object detection is very important and it takes part after applying segmentation using the threshold method with value between 180 and 200 in order to complete segmentation and binarization to extract a brain tumor from MRI image which is clear in the figure 7 . In the three parts of colors red, green, blue the object appears and can observe. Moreover, the goal of this detection is to make notification about object detection on each color and observe the difference as a result the brain tumor appears in the figure 7 .

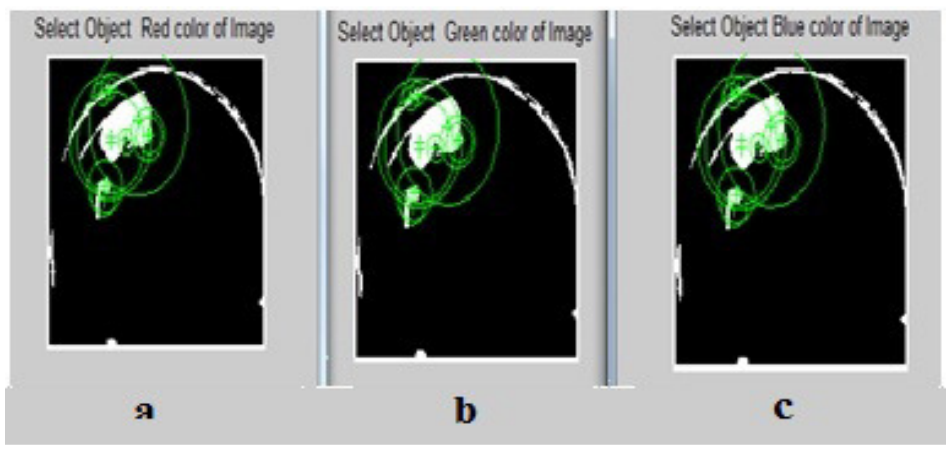

Figure 7. Shows object detection with circle(a) Red (b) Green (c) Blue image.

\subsection{Second experiment.}

It is valuable to test and check multiple brain tumor images, in this paper is tested, checked , verified multiple brain images with different contrast, brightness, noise in order to achieve detection and make the comparisons and get accurate results, This section repeats the previous method and steps which starts with a grayscale image that comes from original MRI color image and its clearly in figure 8 , combines all parts. 

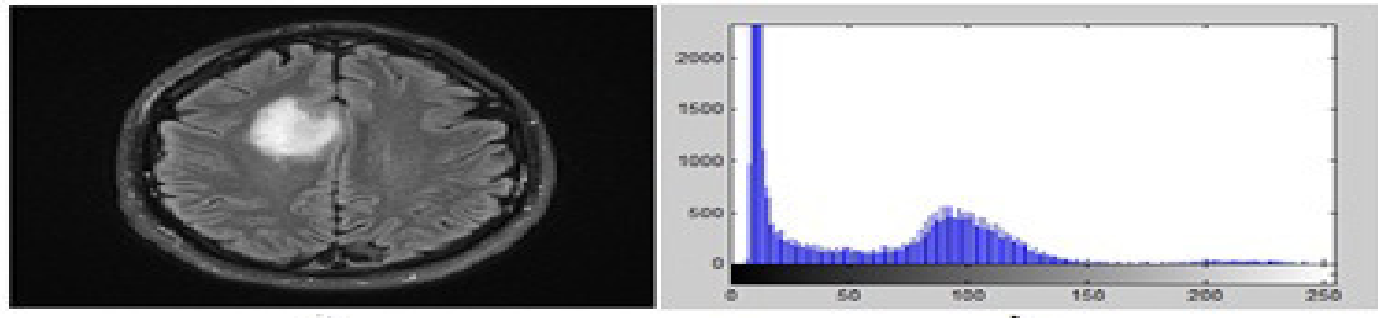

b

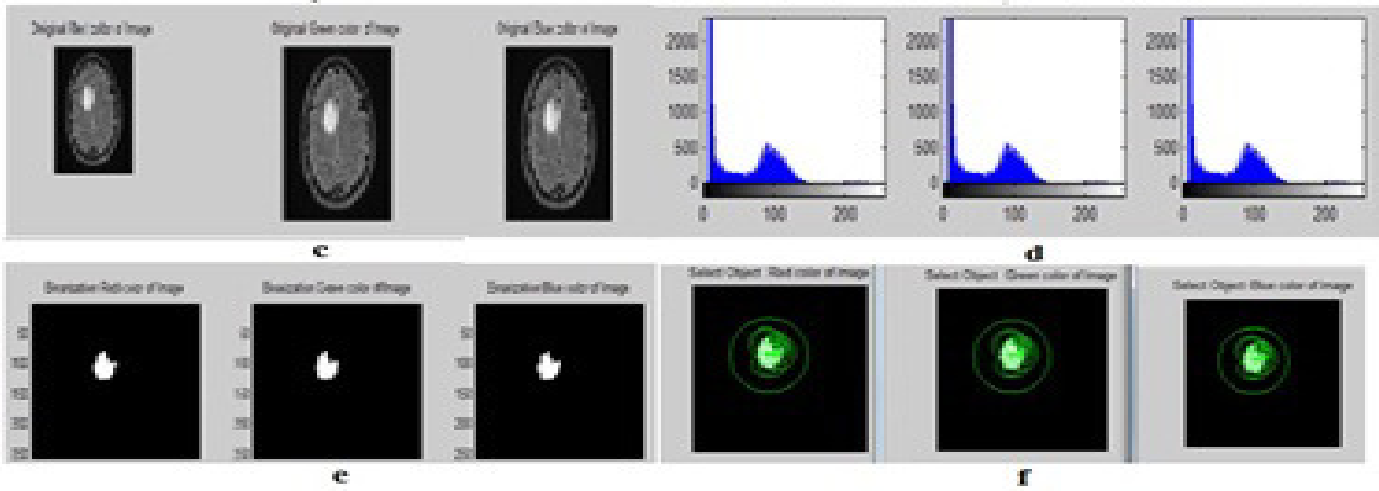

Figure 8. a) Presents gray level information from the original MRI image which captures brain tumor,

b) Presents histogram for the original gray image. c) Separate Red, Green and Blue of gray image,

d) Presents histogram for each Red, Green and Blue image , e) Presents image binarization for each Red, Green and Blue image, f) Presents object detection with circle for each Red, Green and Blue image

\section{DISCUSSION THE RESULTS}

The suggested method is utilized to reduce the process of information needed in the whole brain tumor original image to detect and segment tumor in another image at the beginning of any algorithm, the method proposed dealing directly with the orginal MRI image without need to convert the original image to grayscale image which will lead to reduce the complexity of code and as the result will lead to reduce the time complexity of the process image as well as minimize the information needed by using one color. Moreover, less information and calculation when check one of three color data. The result of the segmentation and binarization when applying on red, green, blue color of the original image is object detection, which makes the comparison more sufficient to prove the results. The proposed method gives very good results for different MRI images.

\section{CONCLUSION}

The results prove that, improve the speed of image processing is possible by separating image colors this paper use medical image, brain tumor as a case study to test object detection and the result will be the same if it process separately this directly will lead to enhance of the whole manipulation in the image. Moreover, processing color image to red or green or blue and working with one color will bring this enhancement because of this extract on color for example Red color from color image directly and working with it is better than change image to gray level, furthermore it is like working with less information, less mathematic operation. The benefit of this work will help online algorithm developers, online manipulation and even in image uploading to test fast algorithms and online application. There is no difference in tumor detection between gray image or using one color channel which is clear when compare histograms and 
objects in this case it is better to use one channel from a color image without changing color image to grayscale for those cases.

\section{ACKNOWLEDGEMENTS}

I would like to express my deepest appreciation to all those who provided me the possibility to complete this research paper.

\section{REFERENCES}

[1] Ruchi D. Deshmukh \& DYPIET Pimpri," Study of Different Brain Tumor MRI Image Segmentation Techniques ", April, 2014, International Journal of Computer Science Engineering and Technology, Vol 4, Issue 4,133-136.

[2] T. Kim \& Ubiquitous," computing and multimedia applications",(2011) Berlin: Springer.

[3] Tai-Hoon Kim \& Debnath Bhattacharyya, (2011) ," Analysis Brain Tumor Detection Using MRI Image", MPCT, Hannam, university, Vol.151,pp 307-314.

[4] Madhurima Banerjee \& Ranjita Chowdhury \& Samir Kumar Bandyopadhyay, (2015), "DETECTION OF BRAIN TUMOR FROM MRI OF BRAIN",Vol. 02, Issue, 12, pp. 1555-1559.

[5] S. Karthigaiselvi and T. Kalaiselvi,(2017),"Investigation of Image Processing Techniques in MRI Based Medical Image Analysis Methods and Validation Parameters for Brain Tumor", Current Medical Imaging Reviews, vol. 13.

[6] N. Ayache \& J.S. Duncan,( 2016), Medical Image Analysis, journals., MICCAI Society .

[7] Pankaj Kr. Saini1\& Mohinder Singh\& Maharishi Ved Vyas,( 2015)," BRAIN TUMOR DETECTION IN MEDICAL IMAGING USING MATLAB", Engineering College Jagadhri, Yamuna Nagar India.

[8] A. Aslam, E. Khan and M. Beg, "Improved Edge Detection Algorithm for Brain Tumor Segmentation", Procedia Computer Science, vol. 58, pp. 430-437, 2015.

[9] Madhurima Banerjee \& Ranjita Chowdhury \& Samir Kumar Bandyopadhyay, (2015), "DETECTION OF BRAIN TUMOR FROM MRI OF BRAIN",Vol. 02, Issue, 12, pp. 1555-1559.

[10] Jian-Jiun Ding \& Cheng-Jin Kuo \& Wen-Chih Hong, "AN EFFICIENT IMAGE SEGMENTATION TECHNIQUE BY FAST SCANNING AND ADAPTIVE MERGING", Graduate Institute of Communication Engineering, National Taiwan University.

[11] N. Menon \& R. Ramakrishnan, 2015 , "Brain Tumor Segmentation in MRI images using unsupervised Artificial Bee Colony algorithm and FCM clustering," 2015 International Conference on Communications and Signal Processing (ICCSP).

[12] P. S. Juhi \& S. S. Kumar, "Bounding box based automatic segmentation of brain tumors using random walker and active contours from brain MRI, ), 2014, " International Conference on Control, Instrumentation, Communication and Computational Technologies (ICCICCT.

\section{AUTHOR}

Ahmed B Salem Salamh

Department of Computer, Zawia University, Zawia City, Libya

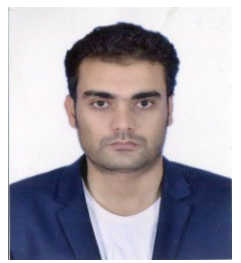

